\title{
Particle Swarm based Unsharp Masking
}

\author{
Dr. S. Mohamed \\ Mansoor Roomi \\ Thiagarajar College of \\ Engineering \\ Thirupparankundram, Madurai \\ Tamil Nadu, India \\ smmroomi@tce.edu
}

\author{
R. Jyothi Priya \\ Thiagarajar College of \\ Engineering \\ Thirupparankundram, Madurai \\ Tamil Nadu, India \\ rjyothipriyaece@gmail.com
}

\author{
S. Yaseer Arafath \\ Thiagarajar College of \\ Engineering \\ Thirupparankundram, Madurai \\ Tamil Nadu, India \\ arabu_7@yahoo.com
}

\author{
S. Gift Rojan \\ Thiagarajar College of \\ Engineering \\ Thirupparankundram, Madurai \\ Tamil Nadu, India \\ rojan.gift@gmail.com
}

\begin{abstract}
Edge enhancement is a predominant process in vision based applications. The performance of the image analysis and interpretation tasks depends on the quality of the image features. It insists that an image should be pre-processed to enhance the fine details like edges. Linear Unsharp Masking (UM) is a conventional method to enhance the edges in the image. The effect of Unsharp Masking depends on the scaling factor provided by the user. In this paper, a novel reference free edge enhancement method called, Particle Swarm based Unsharp Masking (PSUM) is introduced where the scaling factor is optimized through Particle Swarm Optimization by minimizing the blur function without a priori information about the content of the image. The proposed work has been tested over various types of images and low resolution videos and proved to enhance the edges.
\end{abstract}

\section{Keywords}

Unsharp masking, Particle Swarm Optimization, Blur metric, Focus, Edge sharpness

\section{INTRODUCTION}

Images have wide application in remote sensing, medical imaging, surveillance, security and communication. The acquired images are subjected to storage, transmission and process to interpret the objects within them [1]. The visual interpretation of the image depends on the quality of the image. The contrast and fine details determine the visual quality of an image. However, most of the low cost imaging

*Corresponding author

Permission to make digital or hard copies of all or part of this work for personal or classroom use is granted without fee provided that copies are not made or distributed for profit or commercial advantage and that copies bear this notice and the full citation on the first page. To copy otherwise, to republish, to post on servers or to redistribute to lists, requires prior specific permission and/or a fee.

ICVGIP' 10, December 12-15, 2010, Chennai, India

Copyright 2010 ACM 978-1-4503-0060-5/10/12 ...\$10.00. technology produces poor quality images. Therefore, image focusing/sharpening plays a major role in vision based applications. Image quality can be improved by controlling focus of the lens automatically [2]; however, this involves additional hardware. In recent years, image sharpening becomes more important in low resolution image processing such as identification of people in closed-circuit television camera. Generally, the edge sharpening filters are classified into linear and non- linear filters [3]. A classical linear method for edge enhancement is simple unsharp masking (UM). A fraction of the high pass filtered image is added to the original data and the resulting effect produces edge enhancement and noise amplification as well [4]. In order to address this issue, the nonlinear filters are introduced to provide better compromise between the image sharpening and noise attenuation. An order statistical filter, lower-upper-middle (LUM) filter is proposed to smoothen, sharpen and outlier rejection [5]. Weighted median filter (WMF) has been experimented as a replacement for high-pass filters in the UM and also provides outlier suppression [6]. The extension of linear combination of polynomial terms in quadratic volterra(QV) filters [7] with WM, called quadratic weighted median filter $(\mathrm{QWM})$ is derived to yield robust outlier rejection and noise suppression [8]. Morphological filters are also used to detect the edges and sharpen them [9]. The band-pass characteristics of bilateral filters are refined in band pass epsilon filter (BPEF) and used for edge enhancement [10]. An adaptive linear filter based on neural network (NN) is introduced to detect the artifacts, reduce them and enhance the edges [11]. It requires the enormous amount of dataset to train them so that the edge enhancement depends on the content of the training dataset. Most of the filters in literature consist of a lot of user-defined parameters or weights to the pixels which depends on the content of the image. Since the performance of the enhancement depends on such user-specified values, it leads to poor or over enhancement and artifacts on images. Optimization tools like genetic algorithm (GA), particle swarm optimization can play a role in optimizing user specified values to provide the good edge enhancement. The coefficients of weighted vector direction filter (WVDF) [12] are optimized using GA to enhance the 
contrast and detail enhancement [13]. It incorporates the mean absolute error (MAE), requires the original image, for GA optimization. In real time, obtaining the good quality reference image is impossible. Thus the independence of the edge sharpening technique with respect to the original image (No Reference) can make the enhancement process, self evolutionary. In [14], a self-evolutionary Particle Swarm Optimization (PSO) technique is utilized for removing impulse noise. A contrast and detail enhancement algorithm based on optimizing this non-linear problem through PSO has been presented in [15]. The proposed self-evolutionary UM uses PSO to adapt the scaling factor to enhance edges. This performs better than the existing methods available in literature. The rest of the paper is organized as follows. Section 2 describes the proposed PS based unsharp masking (PSUM) which utilizes PSO to obtain an adaptive image enhancement. Section 3 discusses the experimentation details along with results. The concluding remarks are made in Section 4.

\section{PROPOSED METHOD}

In applications such as medical imaging including radiographic and ultrasound images, video surveillance, military surveillance, the captured images are blurred due to either error or, as a natural effect of a particular method of image acquisition, compression and transmission etc. The blur artifact eradication and the fine details enhancement need to be effected to improve the visual quality of the image. Figure 1 shows the schematic of the proposed, such blur reduction/ edge enhancement technique, where the scaling factor of conventional unsharp masking is optimized by Particle Swarm Optimization

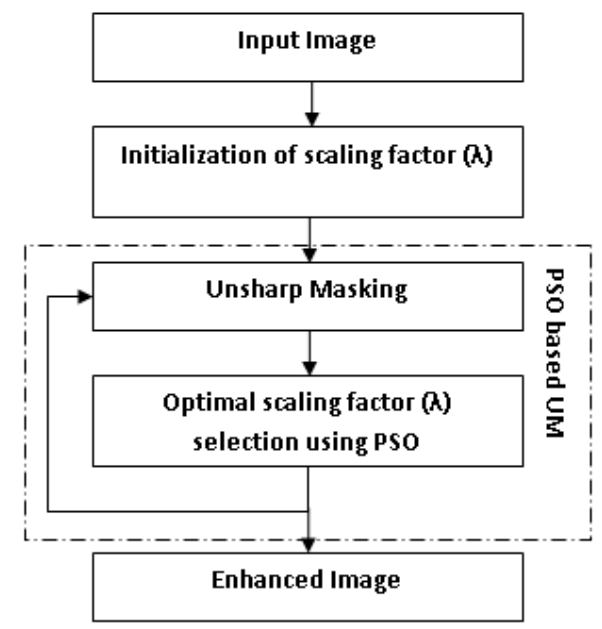

Figure 1: Schematic of the Proposed Method

\subsection{Unsharp Masking (UM)}

Generally in the linear unsharp masking a signal proportional to the unsharp or low-pass filtered version of the image is differenced from the original image to provide edge enhanced image. This is equivalent to adding the gradient or a high-pass signal to the image. This type of enhancement is given by

$$
\hat{x}(m, n)=x(m, n)+\lambda \Delta x(m, n)
$$

where $\hat{x}(m, n)$ is the edge enhanced image, $x(m, n)$ is the original image, $\Delta x(m, n)$ is the correction signal computed as the output of a linear highpass filter and $\lambda$ is the positive scaling factor that controls the level of contrast enhancement achieved at the output.

As mentioned earlier, the performance of the linear UM depends on the high pass filter used. High pass filters like prewitt, sobel can be used to obtain gradient of the image. The high pass filter used for edge enhancement should be immune to noise and provide best edge localization. According to [16], Laplacian of Gaussian (LoG) filter outperforms the other gradient operators. However, the larger scale of variance of the Gaussian leads to the less accurate in the edge localization [17]. Laplacian mask is a second-order derivative, isotropic filter which is rotation invariant to the image. Since Laplacian operator highlights gray-level discontinuities in an image and deemphasizes regions with slowly varying gray levels, it is better for edge localization and immune to small noise level when compared to sobel, prewitt and LoG. The second-order derivative of an image $x(m, n)$ obtained using Laplacian operator with $90^{\circ}$ rotation increment is defined as

$$
\nabla^{2} x=\frac{\partial^{2} x}{\partial m^{2}}+\frac{\partial^{2} x}{\partial n^{2}}
$$

The partial second-order derivative in the m-direction and n-direction is given in equation (3) and (4) respectively.

$$
\begin{gathered}
\frac{\partial^{2} x}{\partial m^{2}}=x(m+1, n)+x(m-1, n)-2 x(m, n) \\
\frac{\partial^{2} x}{\partial n^{2}}=x(m, n+1)+x(m, n-1)-2 x(m, n)
\end{gathered}
$$

Using equations (3) and (4), the implementation of $2-\mathrm{D}$ Laplacian in equation (2) can be represented as

$$
\begin{aligned}
\nabla^{2} x=x(m & +1, n)+x(m-1, n) \\
& +x(m, n+1)+x(m, n-1)-4 x(m, n)
\end{aligned}
$$

Similarly, the mask whose center coefficient is negative with rotation increment of $45^{\circ}$ can be defined as

$$
\begin{gathered}
\nabla^{2} x=x(m-1, n-1)+x(m-1, n)+x(m-1, n+1) \\
+x(m, n-1)+x(m, n+1)+x(m+1, n-1) \\
+x(m+1, n)+x(m+1, n+1)-8 x(m, n)
\end{gathered}
$$

The Laplacian mask, which contains the positive coefficient in the center of the mask, is used for attaining the gradient of the image. The quality of the enhanced image obtained from unsharp masking also depends on the selection of scaling factor $\lambda$. Since the edge sharpening is a content based phenomenon, the scaling factor could not be universally fixed for all images. The random selection of scaling factor $\lambda$ may cause the over or poor enhancement in an image due to the large and small value of $\lambda$. Over enhancement leads to the ringing artifacts and poor enhancement leaves the image smooth. In both cases, the visual quality of an image gets affected. Therefore the optimal selection of scaling factor plays a significant role in edge enhancement. 


\subsection{Optimization of Scaling factor $(\lambda)$ through PSO}

Traditional search methods using Calculus, Enumeration, and Random Walks, fails in many circumstances to find strong solutions. Thus we migrate towards Evolutionary Techniques such as PSO to optimize the scaling factor. Unlike in other evolutionary strategies, PSO has no selection operation. All particles in PSO are kept as members of the population through the course of the run. PSO is the only algorithm that does not implement the survival of the fittest. Particle Swarm Optimization begins with an initial set of random solution. Each potential solution in the set called particle is given a random velocity and is propagated through the problem space. The particles have memory and share information of their previous best position and the over all best position ever traveled by a particle in the swarm. The flow of the PSO based scaling factor selection is illustrated in Figure 2.

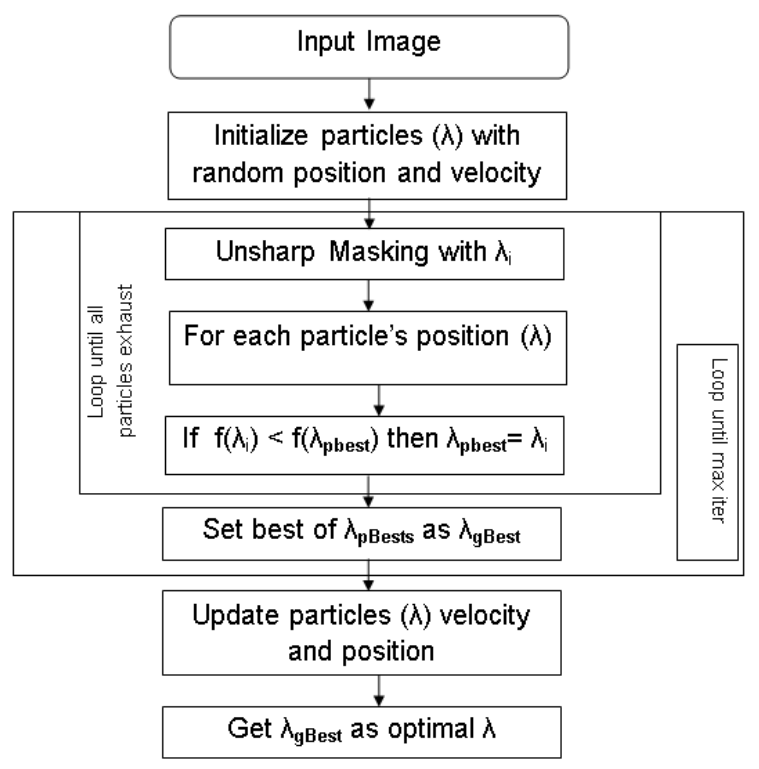

Figure 2: PSO based scaling factor selection

Blur metric, a measure of inhomogenity in an image has been put forward to be used as fitness function to the PSO.

\subsubsection{Fitness Function}

The blur metric, which measures blur present in an image, based on the analysis of the spread of the edges in the image [18]. Since blur metric is a no- reference measurement for edge sharpness, it can be used as self-evaluating objective function in the proposed framework to optimize the scaling factor, $\lambda$. The blur metric computed over an image becomes low when an image is sharpened. Therefore, particle swarm based unsharp masking (PSUM), is achieved by selecting the appropriate and optimal scaling factor $\lambda$, which ultimately tends to reduce the blur metric.

The blur metric, $\eta$, is computed from the width and amount of the edges in the image, obtained through edge detection algorithm. Since the canny operator[19] works good in terms of reduced false detection, edge localization, suppressing multiple edges, it is used to find the edges of an image. Canny edge detector, which is an optimal edge detector among other edge operators, has Gaussian smoothening, zero crossing in first order derivative, edge direction detection, non-maximal suppression and hysteresis based thresholding. The Gaussian smoothening is achieved by convolving the Gaussian kernel with the image is given in equation (7)

$$
y(m, n)=x(m, n) * G(m, n)
$$

where $G(m, n)$ is the Gaussian smoothing kernel, which defined as

$$
G(m, n)=\frac{1}{2 \pi \sigma^{2}} e^{-\frac{m^{2}+n^{2}}{2 \sigma^{2}}}
$$

The horizontal and vertical gradient of the smoothened image is yielded using the sobel operator as given in equation (9) and (10) respectively.

$$
\begin{aligned}
& G_{m}(m, n)=[G(m-1, n-1)+2 G(m-1, n)+G(m-1 . n+1)] \\
& -[G(m+1, n-1)+2 G(m+1, n)+G(m+1, n+1)]
\end{aligned}
$$

$$
\begin{aligned}
& G_{n}(m, n)=[G(m-1, n-1)+2 G(m, n-1)+G(m+1 . n-1)] \\
& -[G(m-1, n+1)+2 G(m, n+1)+G(m+1, n+1)]
\end{aligned}
$$

The magnitude from the gradient along $\mathrm{m}$ - and $\mathrm{n}$ - direction is computed to estimate the strength of the edge as in equation (11)

$$
M a g=\sqrt{G_{m}^{2}+G_{n}^{2}}
$$

The zero crossings corresponding to the first order derivative on the Gaussian smoothened image for reducing the error detection. From $G_{m}$ and $G_{n}$, the direction of the edge is detected by measuring the angle of each edge point as shown in equation (12)

$$
\theta=\tan ^{-1} \frac{G_{n}}{G_{m}}
$$

The edge direction in an image is traced by analyzing the $5 \times 5$ mask of the each gradient pixel and quantized its direction into 5 angles. Then the nonmaximal suppression, is applied on the gradient image, which is used to trace along the edge in the edge direction and suppress any pixel value (denoted as $G_{N}$ )

The hysteresis thresholding is employed to locate the edges from the magnitude of the image. If a single threshold, $T_{1}$ is applied to an image, and an edge has an average strength equal to $T_{1}$, then due to noise, there will be instances where the edge dips below the threshold. Equally it will also extend above the threshold making an discontinuities in edges. To avoid this, hysteresis uses 2 thresholds, a high and a low. Any pixel in the image that has a value greater than $T_{1}$ is presumed to be an edge pixel. Then the any pixels that are connected to this edge pixel and that have a value greater than $T_{2}$ are also selected as edge pixels. The binary edge image $g(m, n)$ is thresholded as in equation (13)

$$
g(m, n)=\left\{\begin{array}{cc}
1, & \text { if } G_{N}(m, n) \geq T_{1} \& G_{N}(m, n) \leq T_{2} \\
0, & \text { else }
\end{array}\right.
$$

The total amount of edges, $\eta_{w}$, in the binary edge image is accumulated as given in equation (14).

$$
\eta_{w}=\sum_{m=0}^{M-1} \sum_{n=0}^{N-1} g(m, n)
$$


where $M \times N$ is the size of the image. Then the edge image is scanned through the horizontal direction and the total number of the edges in the image $\eta_{e}$ is obtained using equation (15)

$$
\eta_{e}=\sum_{i=0}^{M-1} g_{e}(i)
$$

where $g_{e}(i)$ is the no. of edges in $i^{t h}$ row. From the equation (14) and (15), the blur metric $(\eta)$ of an image is calculated as given in equation (16)

$$
\eta=\frac{\eta_{w}}{\eta_{e}}
$$

Since small $\eta$ denotes better edge enhancement in the optimization strategy works around minimizing it with a requisite $\lambda$.

\subsubsection{Algorithm for PSO based $\lambda$ selection}

The algorithm for the PSO based scaling factor selection with minimization of $\eta$ is given below. Initialize the particles i.e., scaling factor $\lambda_{i}$ and the velocity $v_{i}$ for all i Let $\mathrm{n}$ be the size (population) of solution space. Let the fitness function $\mathrm{f}$ be the blur metric as given in equation (16). For every member $1 \leq i \leq n$ of the solution space

- Generate learning factors $\omega, c 1, c 2$ and the random values $b 1, b 2$

-Update the velocity as

$\left.v_{i}=\omega v_{i O l d}+c_{1} b_{1}\left(\lambda_{p B e s t}-\lambda_{i O l d}\right)+c_{2} b_{2}\left(\lambda_{g B e s t}-\lambda_{i O l d}\right)\right)$

-Update the scaling factor as

$$
\lambda_{i}=\lambda_{i O l d}+v_{i}
$$

-When $f\left(\lambda_{i}\right)<f\left(\lambda_{p B e s t}\right)$, update the Individual Best $\left(\lambda_{p \text { Best }}\right)$ for $i$ (particle), the set of scaling factor that yields the best Fitness value minimum Blur metric, $\eta$, as given in equation (19)

$$
\lambda_{p B e s t}=\lambda_{i}
$$

-When $f\left(\lambda_{g B e s t}\right)<f\left(\lambda_{i}\right)$, update the Global Best $\left(\lambda_{g B e s t}\right)$, the scaling factor yields the minimum Blur metric $(\eta)$ in a global sense (i.e.) Best of Individual Best's as shown in equation (20).

$$
\lambda_{g B e s t}=\min \left\{\lambda_{p B e s t s}\right\}
$$

The above algorithm is iterated until

$$
\lambda_{\text {gBestOld }}-\lambda_{\text {gBestOld }} \leq \epsilon
$$

The convergence yields $\lambda_{g B e s t}$, the optimal scaling factor that minimizes blur metric, $\eta$. With the $P_{g B e s t}$ as scaling factor $\lambda$, the enhanced image is obtained as given in equation (1).

\section{RESULTS AND DISCUSSIONS}

The standard optical images like 'lena', 'boat', 'bridge', 'house', SEM image of 'Metal Plate' and satellite image of 'Moon' shown in Figure 3, are used for experimentation. Each image has the size of $(256 \times 256)$ an 8 -bit grayscale image. The test images are subjected to blur by a Gaussian smoothening filter. In this section the visual quality of the enhanced edges used in this paper are described in detail, followed by quantitative measures such as Focus, Blur metric,
Edge sharpness. The effectiveness of the proposed work is illustrated by comparing it with the existing methods which are Weighted Median Filter (WMF) [9], Quadratic Volterra filter (QVF) [6], Quadratic Weighted Median Filter (QWM) [7] and Edge-Detected Guided Morphological Filter (EDMOG) [8].

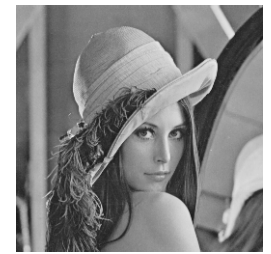

(a) Lena

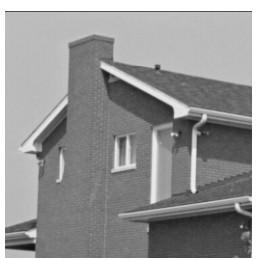

(d) House

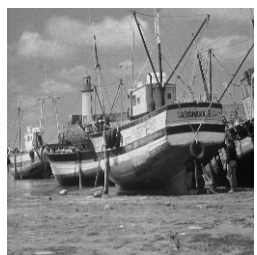

(b) Boat

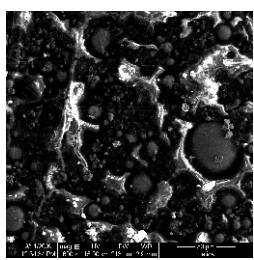

(e) Plate

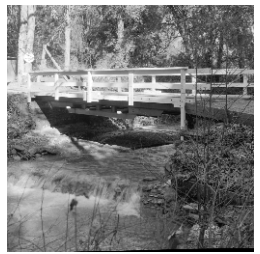

(c) Bridge

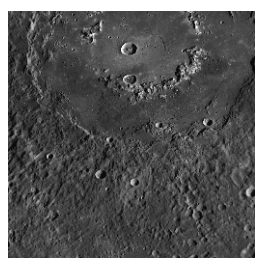

(f) Moon
Figure 3: Sample test images used for Experimentation

\subsection{Qualitative Performance Analysis}

The visual quality of the proposed work is examined through Lena image. Lena image is blurred with Gaussian smoothening mask having the size of $3 \times 3$ and variance of 6 . The blurred image shown in Figure 4 (a) is subjected to various edge enhancement techniques. The resultant of WMF is shown in Figure 4 (b). Since median operator is employed for sharpening, it also provides smoothness in image which can be easily seen in nose of Lena. The result obtained from the QVF is illustrated in Figure 4 (c). It enhances the edges and also maintains other pixel values. By applying the QWM, the edges are enhanced but the non-edge pixels are affected. So the image brightness is reduced. The outcome of the QWM is shown in Figure 4 (d). The EDMOG filter produces the better edge enhancement when compared to the previous methods. However, the dilated effects present in the image, which can be seen in nose of Lena. The resultant of EDMOG is shown in Figure 4 (e). However, the proposed method outperforms the other methods. The proposed PSUM method sharpens the edges and also preserve the brightness and contrast of the image. The enhanced image attained from the PSUM is demonstrated in Figure 4(f). Similarly, the results shown in shown in Figure 5 indicates better qualitative performance of proposed PSUM algorithm.

The efficacy of the proposed work is evaluated through the subjective phenomenon. On the other hand, the robustness of the enhancement algorithm should be validated by the quantitative analysis. 


\subsection{Quantitative Performance Analysis}

The effectiveness of the edge enhancement depends on the sharpened edges, non-smoothness properties of the image. By evaluating the edge sharpness and inhomogeneity measures like Focus $(\zeta)$, Blur metric $(\eta)$ and Edge Sharpness $(\xi)$, the efficiency of an edge enhancement technique can be determined. Following sections briefly describes these measures.

\subsubsection{Focus}

Focus present in an image can be calculated with statistical measures [20]. Let an image be partitioned into nonoverlapping $S$ segments amd each image is partitioned $W$ windows. Let $M\left(W_{k}\right)$ be mean of $k^{t h}$ window and $D\left(W_{k}\right)$ be mean absolute deviation of $k^{t h}$ window then Horizontal Edge Count $\left(\varepsilon_{x}(W)\right)$

$\varepsilon_{x}(W)=\frac{1}{K \times L} \sum_{k=0}^{K-1} \sum_{l=0}^{L-1} \tau(|W(k, l)-W(k, l+1)|, \varrho(W))$

where $\tau$ and $\varrho$ are described below

$$
\begin{gathered}
\tau_{(x, y)}=\left\{\begin{array}{lc}
1, & x>y \\
0, & \text { otherwise }
\end{array}\right. \\
\varrho(W)=\beta * D(W)
\end{gathered}
$$

Similarly Vertical Edge Count $\left(\varepsilon_{y}(W)\right)$ can be calculated. Edge magnitude of an image in horizontal direction is defined as in equation (23)

$$
\psi_{x}(W)=\sum_{k=0}^{K-1} \sum_{l=0}^{L-1} \Upsilon((|W(k, l)-W(k, l+1)|-\varrho(W)))
$$

where

$$
\Upsilon(x)= \begin{cases}x & x>y \\ 0 & x \leq y\end{cases}
$$

Similarly edge magnitude in vertical direction $\psi_{y}(W)$ can be calculated. Then, Normalized Mean Edge Magnitude $\left(\varepsilon_{M}(W)\right)$ is derived as

$$
\varepsilon_{M}(W)=\frac{1}{D(W)}\left(\frac{\psi_{x}(W)+\psi_{y}(W)}{\varepsilon_{x}(W)+\varepsilon_{y}(W)}\right)
$$

After calculating $\varepsilon_{M}(W)$ for all windows within a segment, mean of this measure over the entire segment, denoted as $\varepsilon_{M}(S)$, is also obtained. The focus $(\zeta)$ of an image is obtained using equation (25)

$$
\zeta=1-\frac{1}{S} \sum_{i=0}^{S-1} \varepsilon_{M}\left(S_{i}\right)
$$

\subsubsection{Edge Sharpness}

Edge Sharpness $(\xi)$ is used as one of the evaluation parameter for image sharpness. It is defined by average intensity of the high spatial frequency component in the edge area. By using this, the edge sharpness is measured without modulation transfer function. The edge sharpness (ES) is defined as given in equation (26)

$$
\xi=\frac{\iint_{E(x)}|x(m, n) \otimes s s(m, n)| d m d n}{A_{E}(x)}
$$

where $E(x)$ is edge area in the input image, $A_{E}$ is amount of edge area $E(x)$. The $s s(m, n)$ is a high band-pass filter. The numerator in equation (26) represents integral high spatial frequency components in $E(x)$.

\subsubsection{Quantitative Measures}

The quantitative measure for Lena image is shown in Table I. Large value in the Focus $(\zeta)$, smaller values in blur metric $(\eta)$ and edge sharpness $(\xi)$ shows that the proposed PSUM method enhances the edges better than the other existing method. The quantitative measures for 'Boat', 'Bridge', 'House', 'Metal Plate' and 'Moon' are shown in Table IIVI respectively. The performance of the edge enhancement

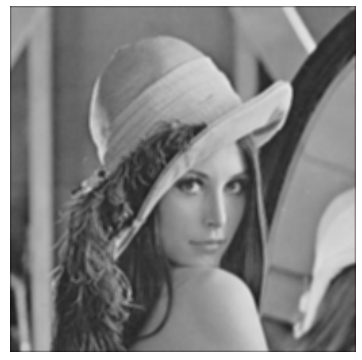

(a) Gaussian Blurred image with $\sigma=6$

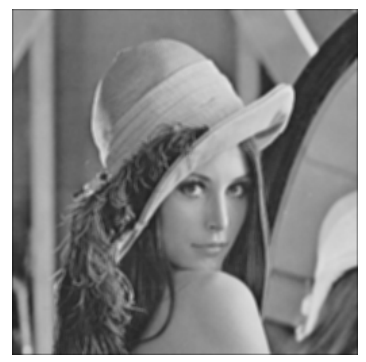

(c) QV Filter

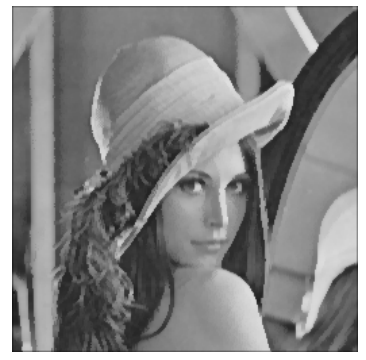

(e) EDMOG Filter

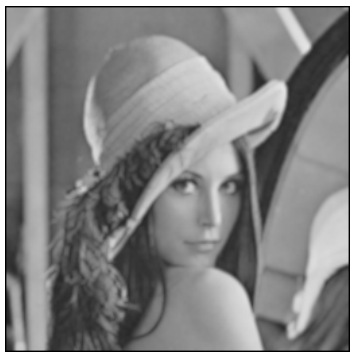

(b) WM Filter

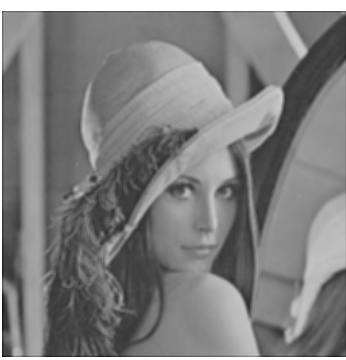

(d) QWM Filter

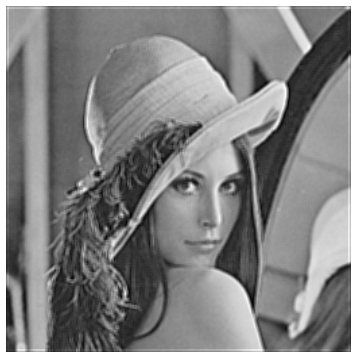

(f) Proposed PSUM
Figure 4: Qualitative Analysis of Proposed with Other Methods

techniques for various smoothening levels is tested for different images. Lena image is utilized to describe the experimentation study. Here, the variance of the Gaussian 
smoothening filter is varied from 0.5 to 6 with the step size of 0.5. The qualitative measures, $\zeta, \eta$ and $\xi$ for various $\sigma$ is illustrated in Figure 6 (a), (b) and (c) respectively. The graphs interprets that the PSUM outperforms others. According to the graphs, EDMOG works well for the very small value of smoothening variance. But the proposed framework dominates from the Gaussian smoothening level of 1 .

Table 1: Quality Measures for Lena Image

\begin{tabular}{|l|l|l|l|}
\hline Methods & $\begin{array}{l}\text { Focus } \\
(\zeta)\end{array}$ & $\begin{array}{l}\text { Blur } \\
\operatorname{metric}(\eta)\end{array}$ & $\begin{array}{l}\text { Edge } \\
\text { Sharpness }(\xi)\end{array}$ \\
\hline Blurred $(\sigma=6)$ & 0.9245 & 6.7554 & 15.382 \\
WMF & 0.9279 & 1.2537 & 15.5708 \\
QV & 0.9245 & 1.2317 & 15.382 \\
QWM & 0.9071 & 1.2184 & 51.8219 \\
EDMOG & 0.9284 & 5.0634 & 10.553 \\
PSUM & $\mathbf{0 . 9 3 6 1}$ & $\mathbf{1 . 1 3 7 4}$ & $\mathbf{2 . 7 4 7 3}$ \\
\hline
\end{tabular}

Table 2: Quality Measures for Boat Image

\begin{tabular}{|l|l|l|l|}
\hline Methods & $\begin{array}{l}\text { Focus } \\
(\zeta)\end{array}$ & $\begin{array}{l}\text { Blur } \\
\operatorname{metric}(\eta)\end{array}$ & $\begin{array}{l}\text { Edge } \\
\text { Sharpness }(\xi)\end{array}$ \\
\hline Blurred $(\sigma=6)$ & 0.9205 & 1.7052 & 6.3416 \\
WMF & 0.9227 & 1.6903 & 6.5978 \\
QV & 0.9205 & 1.7052 & 6.3416 \\
QWM & 0.9065 & 1.6934 & 61.7602 \\
EDMOG & 0.9238 & 1.6498 & 6.0898 \\
PSUM & $\mathbf{0 . 9 5 4 5}$ & $\mathbf{1 . 6 2 6 7}$ & $\mathbf{4 . 7 6 7 4}$ \\
\hline
\end{tabular}

Table 3: Quality Measures for Bridge Image

\begin{tabular}{|l|l|l|l|}
\hline Methods & $\begin{array}{l}\text { Focus } \\
(\zeta)\end{array}$ & $\begin{array}{l}\text { Blur } \\
\operatorname{metric}(\eta)\end{array}$ & $\begin{array}{l}\text { Edge } \\
\text { Sharpness }(\xi)\end{array}$ \\
\hline Blurred $(\sigma=4)$ & 0.9493 & 1.7401 & 6.339 \\
WMF & 0.9505 & 1.808 & 6.597 \\
QV & 0.9493 & 1.7401 & 6.339 \\
QWM & 0.9381 & 1.7101 & 62.3154 \\
EDMOG & 0.9526 & 1.6641 & 6.0762 \\
PSUM & $\mathbf{0 . 9 7 9 4}$ & $\mathbf{1 . 6 3 3 1}$ & $\mathbf{1 . 2 6 2 2}$ \\
\hline
\end{tabular}

Table 4: Quality Measures for House Image

\begin{tabular}{|l|l|l|l|}
\hline Methods & $\begin{array}{l}\text { Focus } \\
(\zeta)\end{array}$ & $\begin{array}{l}\text { Blur } \\
\operatorname{metric}(\eta)\end{array}$ & $\begin{array}{l}\text { Edge } \\
\text { Sharpness }(\xi)\end{array}$ \\
\hline Blurred $(\sigma=4)$ & 0.7206 & 1.826 & 22.122 \\
WMF & 0.7033 & 1.8113 & 18.7946 \\
QV & 0.7206 & 1.826 & 22.122 \\
QWM & 0.6749 & 1.853 & 89.3594 \\
EDMOG & 0.7319 & 1.7507 & 20.9863 \\
PSUM & $\mathbf{0 . 8 3 7 9}$ & $\mathbf{1 . 7 2 7 5}$ & $\mathbf{1 2 . 0 0 0 6}$ \\
\hline
\end{tabular}

The proposed method has been applied and found to perform well over a real image (Figure 7) with an unknown blur level. Table 7 provides the convergence rate of proposed and other methods on comparisons for 'cameraman' image, the PSUM method converges quickly the existing GA
Table 5: Quality Measures for SEM Image

\begin{tabular}{|l|l|l|l|}
\hline Methods & $\begin{array}{l}\text { Focus } \\
(\zeta)\end{array}$ & $\begin{array}{l}\text { Blur } \\
\operatorname{metric}(\eta)\end{array}$ & $\begin{array}{l}\text { Edge } \\
\text { Sharpness }(\xi)\end{array}$ \\
\hline Blurred $(\sigma=2)$ & 0.9567 & 1.2158 & 143.7208 \\
WMF & 0.9612 & 1.2295 & 81.9878 \\
QV & 0.9567 & 1.2158 & 143.7189 \\
QWM & 0.9469 & 1.2199 & 340.7023 \\
EDMOG & 0.9603 & 1.2045 & 107.2089 \\
PSUM & $\mathbf{0 . 9 7 8 5}$ & $\mathbf{1 . 1 7 1 2}$ & $\mathbf{7 4 . 5 7 1 6}$ \\
\hline
\end{tabular}

Table 6: Quality Measures for Planet Image

\begin{tabular}{|l|l|l|l|}
\hline Methods & $\begin{array}{l}\text { Focus } \\
(\zeta)\end{array}$ & $\begin{array}{l}\text { Blur } \\
\operatorname{metric}(\eta)\end{array}$ & $\begin{array}{l}\text { Edge } \\
\text { Sharpness }(\xi)\end{array}$ \\
\hline Blurred $(\sigma=2)$ & 0.9114 & 1.3828 & 0.1879 \\
WMF & 0.9150 & 1.3949 & 0.2102 \\
QV & 0.9114 & 1.3828 & 0.1879 \\
QWM & 0.8983 & 1.3956 & 1.6925 \\
EDMOG & 0.9204 & $\mathbf{1 . 3 5 2}$ & 0.1682 \\
PSUM & $\mathbf{0 . 9 4 8 7}$ & 1.3584 & $\mathbf{0 . 1 1 1 7}$ \\
\hline
\end{tabular}

Table 7: Convergence rate of PSUM vs Existing methods in [21] for 'Cameraman' Image

\begin{tabular}{|l|l|}
\hline Methods & Convergence \\
\hline GA in [21] & 32 \\
PSO in [21] & 28 \\
Proposed PSUM & 21 \\
\hline
\end{tabular}

and PSO based methods. The computational complexity of the proposed PSUM relies on the velocity and position update (equation (17) and (18) ) is of the order $O(n), 35$. The entire algorithm consumes 10.32 s to enhance a $256 \times 256,8$ bit grayscale image on $\mathrm{P} 4$ computing system. The proposed work has also extended into the video sequences. Here the low resolution Charlie Chaplin movie sequences is taken for experimentation. Each frame of the size $256 \times 256$ is subjected to enhancement by the proposed method. The input frame (frame no.150) and enhanced by PSUM is shown in Figure 8.

\section{CONCLUSIONS}

In this framework, algorithm for edge enhancement is proposed. The optimal scaling factor in the unsharp masking is selected by minimizing the blur metric. Since the blur metric is the measure of in homogeneity in the image, edges can be sharpened in optimal way. The population based PSO provides the best optimal solution quickly. The comparison of quality measures among proposed and other existing methods illustrates that the proposed PSUM yields better edge enhancement by measuring Focus, Blur metric and Edge sharpness. The algorithm is also extended to the low resolution videos. However, the proposed algorithm suffers in the presence of noise due to the usage of the Laplacian operator. Noise suppression and edge enhancement can be achieved through the multi-objective PSO, which is the future direction of this work. 


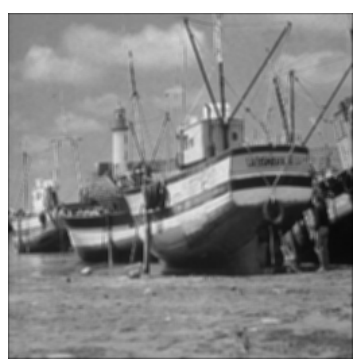

(a)

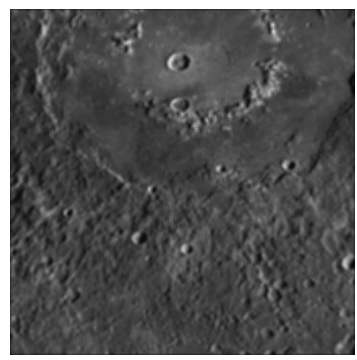

(c)

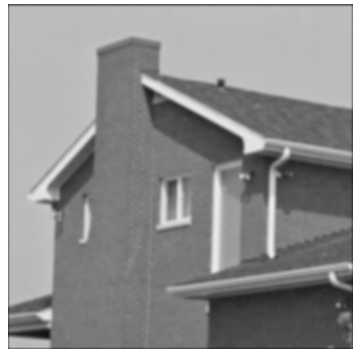

(e)

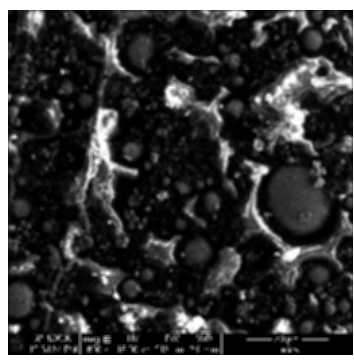

(g)

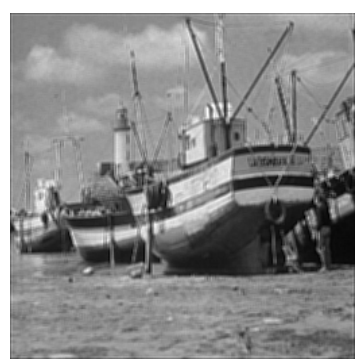

(b)

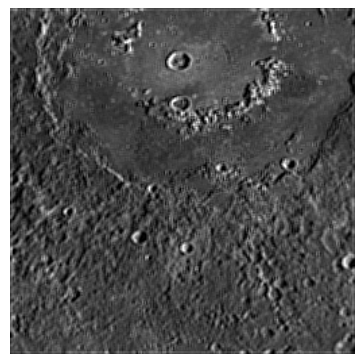

(d)

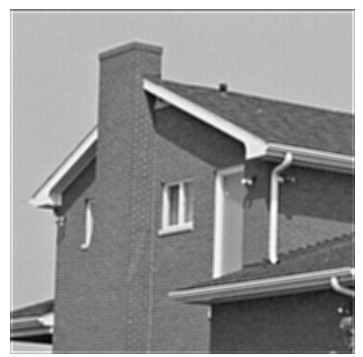

(f)

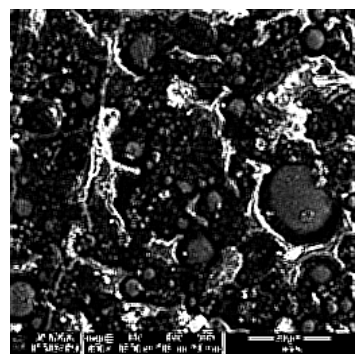

(h)
Figure 5: Results of PSUM for various smoothening level - First row - Smoothened image; Second row - Enhanced image using PSUM; a) Boat $\sigma=5$, b) Planet $\sigma=4$, c) House $\sigma=03$, d) Plate $\sigma=2$, e) - h) Enhanced image of a) - d) using PSUM respectively.

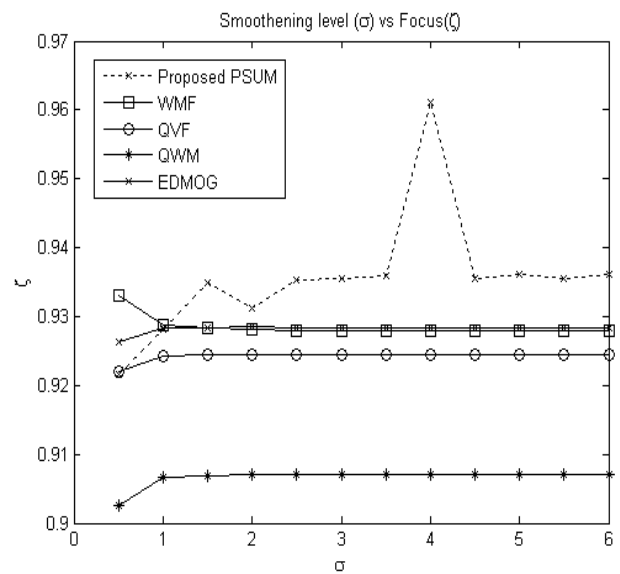

(a)

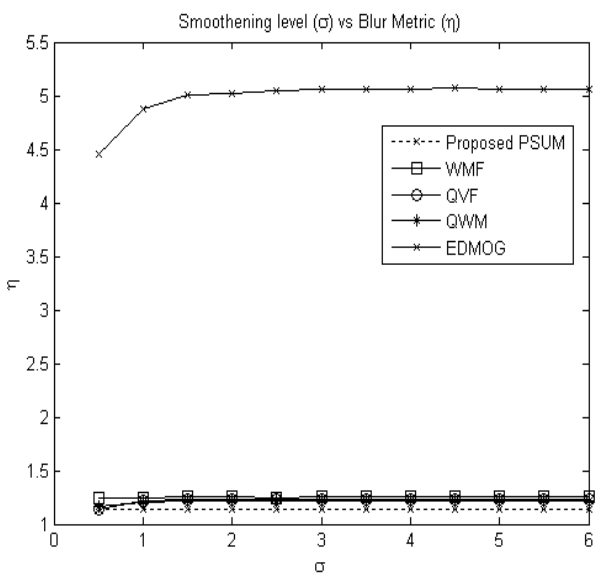

(b)

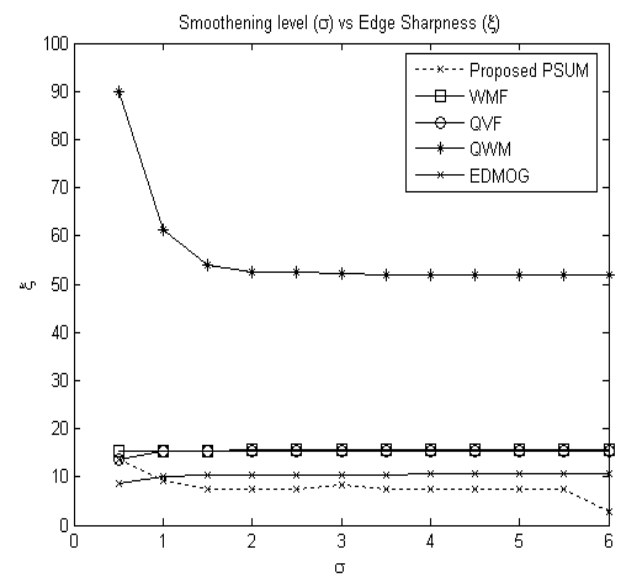

(c)

Figure 6: Performance analysis of proposed method for various Gaussian smoothing variances compared with other methods. (a) Comparison of Smoothing variance vs Focus (b) Comparison of Smoothing variance vs Blur metric (c) Comparison of Smoothing variance vs Edge Sharpness. 


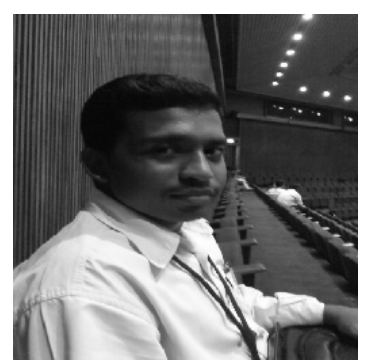

(a)

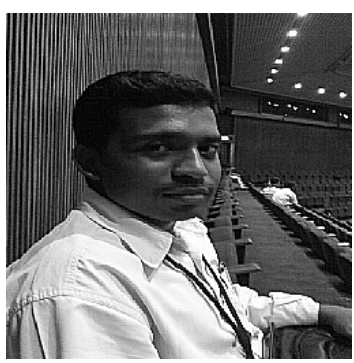

(b)
Figure 7: a) Original Image - Unknown smoothen level b) Enhanced Image using PSUM

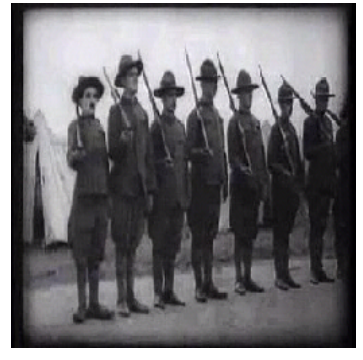

(a)

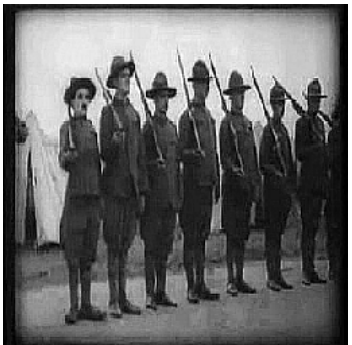

(b)
Figure 8: Video enhancements using proposed method. (a) Original frame (b) Enhanced frame using PSUM

\section{REFERENCES}

[1] W. K. Pratt. Digital Image Processing. John Wiley and Sons, New York, USA, 1978.

[2] E. Krotkov. Focusing. International Journal of Computer Vision, 1(3):223-237, October, 1987.

[3] Fabrizio Russo. Piecewise Linear Model-Based Image Enhancement. EURASIP Journal on Applied Signal Processing, 2004:12,1861-1869.

[4] R.C.Gonzalaz. Digital Image Processing. Prethall, 2004

[5] Russell C. Hardie, Charless G. Boncelet. LUM Filters: A Class of Rank-Order-Based Filters for Smoothing and Sharpening. IEEE Transaction on Signal Processing, 41(3), March 1993.

[6] M. Fischer, J. L. Paredes, and G. R. Arce. Weighted median image sharpeners for the World Wide Web. IEEE Trans. Image Processing, 11(7):717-727, 2002.

[7] Stefan Thurnhofer, Sanjit K. Mitra. A General Framework for Quadratic Volterra Filters for Edge Enhancement. IEEE Transaction on Image Processing, 5(6), June 1996.

[8] Tuncer Can Aysal, Kenneth E. Barner. Quadratic Weighted Median Filters for Edge Enhancement of Noisy Images. IEEE Transaction on Image Processing, 15(11), November 2006.
[9] T. A. Mahmoud and S. Marshall. Edge-Detected Guided Morphological Filter for Image Sharpening. EURASIP JIVP, Vol. 2008, Article ID 970353, 9 pages, doi:10.1155/2008/970353.

[10] Mitshuharu Matsumoto, Shuji Hashimoto. Noise Reduction and Edge Enhancement Based on Band-Pass Epsilon-Filter. International Conference on Information Management and Engineering, 2009.

[11] Hyung-Seung Lee, Rae-Hong Park, SungHee Kim. Adaptive Video Enhancement using Neural Network. IEEE Transaction on Consumer Electronics, 55(3), Aug, 2009.

[12] R. Lukac, B. Smolka, K.N. Plataniotis, A.N. Vene-tsanopulos. Selection weighted vector directional filters. Computer vision and image understanding, Academic press, Vol.94, No. 1-3, 140-167, 2004.

[13] Mantas Paulinas, Andrius Usinskas. A Study of Genetic Algorithms Applications for Image Enhancement and Segmentation. Information Technology and Control, 36(3):278-284, 2007

[14] S. Mohamed Mansoor Roomi, P.L. Muthu Karuppi, P. Rajesh, B. Guru Revathi. A Particle Swarm Optimization Based Edge Preserving Impulse Noise Filter. International Journal of Computer Science, 6(9):1014-1020, 2010

[15] Malik Braik, Alaa Sheta, Aladdin Ayesh. Particle swarm optimisation enhancement approach for improving image quality. International Journal of Innovative Computing and Applications, 1(2):138-145, 2007

[16] Mamta Juneja, Parvinder Singh Sandhu. Performance Evaluation of Edge Detection Techniques for Images in Spatial Domain. International Journal of Computer Theory and Engineering, Vol. 1, No. 5, December 2009.

[17] Raman Maini, Dr. Himanshu Aggarwal. Study and Comparison of Various Image Edge Detection Techniques. International Journal of Image Processing, 3(1), 2009

[18] P. Ma1rziliano, F. Dufaux, S. Winkler, T. Ebrahimi. A no-reference perceptual Blur metric. Proceedings of the International Conference on Image Processing, 3: 57-60,2002.

[19] J.Canny. A Computational Approach to Edge Detection. IEEE Transaction on PAMI,8(6), November 1986.

[20] S. Mohamed Mansoor Roomi, V. Abhai Kumar. Contrast Enhancement Technique based on Visual Significance. International Journal of IISc, 72(2), March, 1999.

[21] Malik Braik, Alaa Sheta, Aladdin Ayesh, Image Enhancement Using Particle Swarm Optimization, Proceedings of the World Congress on Engineering (WCE)1, July, 2007 